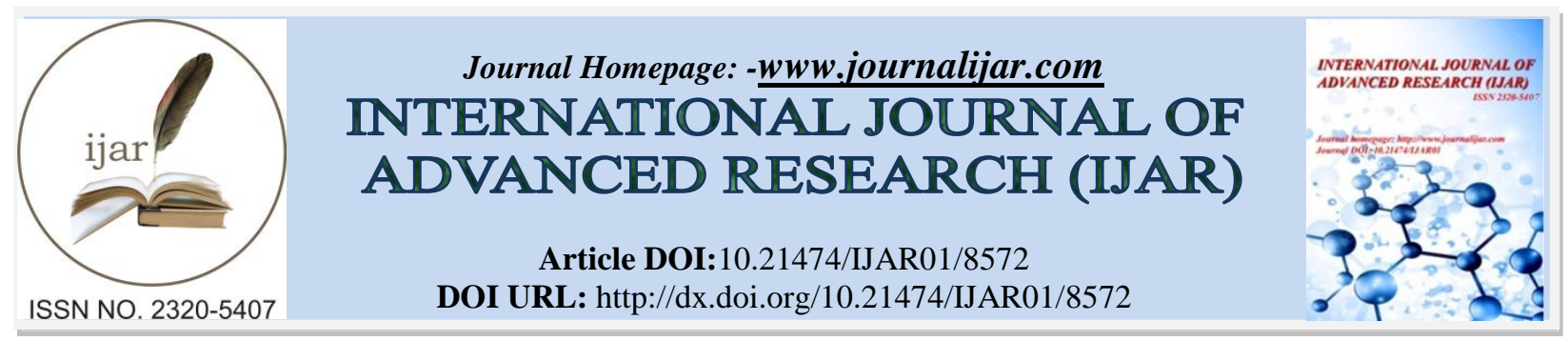

RESEARCH ARTICLE

\title{
DISTRIBUTION PATTERN AND ECO-PHENOLOGICAL STUDIES WITH MEDICINAL VALUE OF PIPER LONGUM L.
}

\section{D. Bhatt.}

Department of Botany, Siddhanath Science Campus, Tribhuvan University, Mahendranagar, Nepal.

\section{Manuscript Info}

Manuscript History

Received: 14 December 2018

Final Accepted: 16 January 2019

Published: February 2019

Key words:-

Composition; Density; IVI; Dominant; Medicinal value.

\section{Abstract}

The study was conducted in three different sites i.e. site 1 , site 2 and site 3. In the present research work, a total number of 67 plant species associated with Piper longum were recorded in different sites. The total density of plant species was 144.25 plant species $\mathrm{m}^{-2}$ at site $1,156.3$ plant species $\mathrm{m}^{-2}$ at site 2 and 237.6 plant species $\mathrm{m}^{-2}$ at site 3 . The density of species associated with Piper at site 3 (237.64) revealed that it had more plant species than that of site 1 and site 2. The maximum density at site 1 was contributed by Imperata cylindrica (15.67 individuals $\mathrm{m}^{-2}$ ) and minimum was that of Azadirachta indica, Dalbergia sisso and Morus alba having 0.33 individuals $\mathrm{m}^{-2}$ whereas at site 2, the maximum density was of Commelina paludosa (12.67 individuals $\mathrm{m}^{-2}$ ) and minimum of Azadirachta indica, (0.33 individuals $\mathrm{m}^{-2}$ ) and at site 3 maximum density was contributed by Parthenium hysterophorus (16.67 individuals $\mathrm{m}^{-2}$ ) and minimum was by Acacia catechu $\left(0.32\right.$ individuals $\left.\mathrm{m}^{-2}\right)$. The mean height of Piper was recorded $101 \mathrm{~cm}$ to $108 \mathrm{~cm}$ with well drained sandy soil of $\mathrm{p}^{\mathrm{H}}$ ranged 5.8 to 7.6. Similarly, the maximum and minimum IVI was occupied by Cynodon dactylon (24.49) and Acacia catechu (3.95) species respectively at site 1, the maximum and minimum IVI was occupied by Cynodon dactylon (23.11) and Chenopodium ficifolium (4.01) and Azadirachta indica (4.01) species respectively at site 2 , and the maximum and minimum IVI was occupied by Imperata cylindrica (22.87) and Acacia catechu (3.39) species, respectively at site 3. The plant has been used traditionally to cure different severe diseases and uplifts the economic status of many local people.

Copy Right, IJAR, 2019,. All rights reserve

\section{Introduction:-}

Nepal lies on the southern slope of central Himalayas and occupies a total area of 1,47,181 sq. $\mathrm{km}$. between the latitudes $26^{\circ} 22^{\prime}$ and $30^{\circ} 27^{\prime} \mathrm{N}$ and the $80^{\circ} 40^{\prime}$ and $80^{\circ} 12^{\prime} \mathrm{E}$. The average length of the country is $885 \mathrm{~km}$ east to west and the width varies $145 \mathrm{~km}-241 \mathrm{~km}$ from north to south. About $86 \%$ of the total land area is covered by hills and high mountains, and the remaining $14 \%$ are flat lands of Terai. The country is extremely rich in terms of floral diversity in proportion to its size. Nepal comprises only $0.09 \%$ of the total land area on global scale but it possesses $2.7 \%$ of the world's recorded flowering plants. A total of 5,856 species of flowering plants, 28 species of gymnosperms, 853 species of bryophytes and 380 species of pteridophytes have been recorded from Nepal (Wilson,

Corresponding Author: - M. D. Bhatt.

Address: - Department of Botany, Siddhanath Science Campus, Tribhuvan University,

Mahendranagar, Nepal. 
1998, 1992; Akiyama et al., 1998 and Koba et al, 1994). The great range of biochemical variation forms tropical to alpine zones brings richness in biodiversity in Nepal.

The vegetation of Nepal classified on the basis of bioclimatic variation by Stearn (1960), Banaergi (1963). Ecologically, Stainton (1972) and Dobremez (1976) divide Nepal in to six bioclimatic zones. Nepal, the Himalayan country is rich in medicinal plant resources. Himalayan medicinal plants are very popular since the Vedic period. The detailed study of all plants is still unknown. Although the enumeration, morphology, distribution and medicinal uses are known but chemical aspect of economically important is still behind.

Herbal medicine is an age-old practice. Nonetheless, with only $10 \%$ of the earth is estimated 2, 50,000 species of higher plants considered medicinal and the lower plants still unexplored. Plants will continue to be the sleeping giant of drug development and serve mankind in the days to come. Plants and their products are important resources for the development of country but peoples are involving in commercial exploitation. In order to protect the medicinal plants of Nepal, it is necessary to first identify them, study their natural distribution, assess their population status and then to take scientific measures to ensure their conservation and cultivation. Due to over use of forested areas and medicinal plants, some plants species have already become rare, endangered, threatened and meet the criteria for red data listing.

There are several medicinal plant species which are widely used by local people in hilly and rural areas of which the species Piper longum is of South Asian origin. Piper longum is commonly found in tropical humid moist places (Sumy et al., 2000), slender aromatic climbing shrubs with perennial woody roots where relative humidity is high (Kritikar \& Basu, 1980; Rastogi \& Malhotra, 1993). Flowers grow in solitary spikes (Rastogi \& Malhotra, 1993, Banerjee et al., 1999; Viswananthan, 1995). The fruit contains a large number of alkaloids and related compounds, the most abundant of which is piperine, followed by methyl piperine, pipernonaline, piperettine, asarinine, pellitorine, piperundecalidine, piperlongumine, piper longuminine, etc (Kritikar and Basu, 1980). Piper longum cures cough, leprosy, diabetes, piles, colic indigestion, anaemia, thirst and dispels cardiac and spleen disorders, chronic fever and loss of appetite. Dried ripe fruits are official parts (Viswananthan, 1995). It is also used as antidote to snake bite and scorpion-sting (Sumy et al., 2000). Clinical studies have revealed that Piper is very effective in treatment of bronchial asthma in children (Dahanukar et al., 1984; Anshuman et al., 1984). The plant Piper longum is frequently found in natural habitats of Far Western Development Region of Nepal. This ecological study provides the understanding of species habitat, relationship, and present status of distribution, economic values, medicinal importance and commercial exploitation. Therefore, the present study was focused on detailed study of Piper longum as well as public attitude and participation in Kanchanpur to conserve biodiversity.

\section{Materials and Methods:-}

Geographically Far Western Development Region lies between $28^{0} 31^{\prime}$ to $30^{\circ} 12^{\prime}$ North latitudes and $80^{\circ} 4^{\prime}$ to $81^{0}$ $45^{\prime}$ East longitudes. The altitude varies from 176 to 7134 above the mean sea level (amsl) and region covers an area of $19,539 \mathrm{~km}^{2}$, which is about $13.3 \%$ of the area of the country (Bhatt et al., 2007). The sites selected for the present study were located at $28^{\circ} 32^{\prime}$ to $29^{\circ} 28^{\prime} \mathrm{N}$ latitudes and $80^{\circ} 03^{\prime}$ to $80^{\circ} 33^{\prime}$ ' E longitudes with an altitudinal range of 76 to 500 amsl of Far Western Nepal.

The study was carried out during July, 2016 to July, 2018 by systematic visits of the study site i.e. site 1 (Jhalari), site 2 (Brahmdev) and site 3 (Bagphanta) of Kanchanpur district at definite regular interval of a month. During the visits, the natural habit growth form, phenology, composition of plant species with Piper and present status of the Piper was determined by visual observation. Plant samples and seeds were collected for morphological and ecological studies. The sampled plants were analyzed for quantitative characters like plant height, leaf area, ratio of leaf length and breath, circumference of stem etc. Quadrates of $1 \times 1 \mathrm{~m}$ size were kept randomly in the study sites and listed the plant species. In each stands, three replicate was executed using the appropriate size of quadrates and their density, abundance, frequency, and important value index (IVI) were calculated as per Misra, 1968, Zobel et al. 1987, Curtis and McIntosh, 1951. The species with higher IVI value were considered as dominant species with Piper. Collected plant species were identified with the help of relevant literatures. Seed shape and colour was calculated from the average of 10 seeds of individual plants. The seed size of Piper longum was calculated as per the procedure in Zobel et al. (1987). The seed output was calculated as per the procedure given by Salisbury (1942). 


\section{Results:-}

\section{Distribution Pattern of Plant Species}

Of the 67 species of plants recorded in the three sites of Kanchanpur, 36 species were found at site 1, 37 species at site 2 and 62 species at site 3 (Table 1). Out of the total plant species, the highest numbers of plant species were recorded at site 3. The common plant species recorded at all sites in association with Piper longum were Achyaranthus aspera, Ageratum conyzoids, Azadirachta indica, Blumea aromatica, Brachieria ramosa, Cassia tora, Chenpodium ficifolium, Cissampelos pareira, Clerodendrum indicum, Commelina benghalensis, Commelina paludosa, Cynodon dactylon, Dalbergia sisso, Desmodium triflorum, Euphorbia hirta, Dryopteris spp, Imperata cylindrica, Justicia procumbens, Lantana camara, Oxalis corniculata and Solanum nigrum.

The percentage wise distribution of plant species at site 1 were $69.4 \%$ dicots species, $25 \%$ monocot species, and $5.6 \%$ pteriodophytes. Similarly, there were $70.3 \%$ dicot species, $24.3 \%$ monocot species and $5.4 \%$ pteridophytes at site 2 and at site $3,72.6 \%$ dicots $34.2 \%$ monocot species and 3.2\% pteridophytes were recorded (Fig. 1).

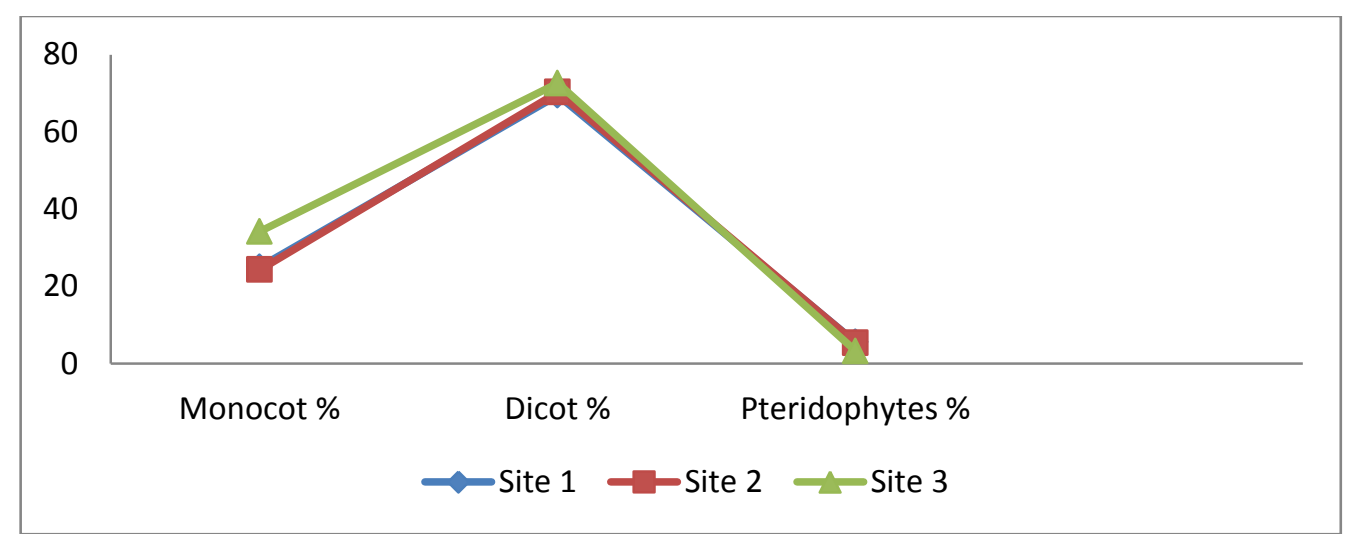

Fig. 1:-Analysis of plant groups in different study sites

The reported species belonged to 34 families: Poaceae/Graminae (11), Asteraceae/Compositae (9). Amaranthaceae (5), Leguminosae (4), Cyperaceae (2), Euphorbiaceae (2), Commelinaceae (2), Acanthaceae (2), Meliaceae (2), Fabaceae (2), Scrophulariaceae (2), Verbenaceae (2) and one species each belonged to Apiaceae, Convolvulaceae, Liliaceae, Asclepidaceae, Umbelliferae, Menispermacae, Labiatae, Diocoreaceae, Dryopteridiaceae, Convolvulaceae, Moaraceae, Marsilaceae, Malvaceae, Myrtaceae, Oxalidaceae, Onagraceae, Papaveraceae, Portulaceae, Polygonaceae, Primulaceae, Rhamnaceae, Rutaceae, Solanaceae and Verbenaceae including Piperaceae of Piper longum (Table 2).

Table 1:-List of plant species associated with Piper longum at the different sites of Kanchanpur $(+=$ Present, $-=$ absent).

\begin{tabular}{|c|c|c|c|c|c|c|}
\hline \multirow[t]{2}{*}{ S.No. } & \multirow[t]{2}{*}{ Name of plant species } & \multirow[t]{2}{*}{ Local name } & \multirow[t]{2}{*}{ Family } & \multicolumn{3}{|c|}{ Distribution of species } \\
\hline & & & & Site 1 & Site 2 & Site 3 \\
\hline 1. & Acacia catechu (L.) Willd ${ }^{\#}$ & Khayer & Leguminosae & + & - & + \\
\hline 2. & Achyranthes aspera L. ${ }^{\dagger}$ & Datiwan & Amaranthaceae & + & + & + \\
\hline 3. & Ageratum conyzoids $\mathrm{L}^{\dagger}{ }^{\dagger}$ & Ganey ghans & Compositae & + & + & + \\
\hline 4. & Alternanthera sessilis (L.) DC. ${ }^{* \#}$ & Bhiringi jahar & Amaranthaceae & - & - & + \\
\hline 5. & Alysicarpus vaginalis (L.) DC. ${ }^{* \#}$ & Chandre ghans & Leguminaceae & - & - & + \\
\hline 6. & Amaranthus spinosus L. ${ }^{*}$ & Kande lunde & Amaranthaceae & - & + & + \\
\hline 7. & Amaranthus viridus $\mathrm{L} .{ }^{* \#}$ & Seto lunde & Amaranthaceae & - & - & + \\
\hline 8. & Argemone mexicana $\mathrm{L} .{ }^{*}$ & Kande & Papaveraceae & - & + & + \\
\hline 9. & Asparagus recemosus Willd $^{\#}$ & Kurilo & Liliaceae & + & - & + \\
\hline 10. & Azadirachta indica (Aiton) Dryand. & Neem & Meliaceae & + & + & + \\
\hline 11. & Blumea aromatica L. ${ }^{\dagger}$ & Bisaune jhar & Asteraceae & + & + & + \\
\hline 12. & Brachieria ramosa (L.) Stapf. ${ }^{\dagger}$ & Likhe banso & Poaceae & + & + & + \\
\hline 13. & Calotropis procera Juss. ${ }^{* \#}$ & Aankh & Asclepiadaceae & - & - & + \\
\hline
\end{tabular}




\begin{tabular}{|c|c|c|c|c|c|c|}
\hline 14. & Cassia tora $\mathrm{L}^{\dagger}$ & Khinkhine & Fabaceae & + & + & + \\
\hline 15. & Centella asiatica (L.) Urban. ${ }^{*}$ & Ghodtapre & Umbelliferae & - & + & + \\
\hline 16. & Chenopodium album $\mathrm{L}^{\dagger}$ & Bethua & Amaranthaceae & + & + & + \\
\hline 17. & Cisssampelos pareira $\mathrm{L}^{\dagger}$ & Musebeli & Menispermaceae & + & + & + \\
\hline 18. & Clerodendrum indicum $(\mathrm{L})$ Kuntce. ${ }^{\dagger}$ & Eklebir & Labiatae & + & + & + \\
\hline 19. & Commelina benghalensis $(\mathrm{L})^{\dagger}$ & Kaane jhar & Commelinaceae & + & + & + \\
\hline 20. & Commelina paludosa Blume $^{\dagger}$ & Kaane jahar & Commelinaceae & + & + & + \\
\hline 21. & Cynodon dactylon $(\mathrm{L})$ Pers. $^{\dagger}$ & Dubo & Gramineae & + & + & + \\
\hline 22. & Cyperus esculentus L. ${ }^{\#}$ & Mothe & Cyperaceae & + & - & + \\
\hline 23. & Cyperus rotundus $\mathrm{L}^{\dagger}$ & Mothe & Cyperacera & + & + & + \\
\hline 24. & $\begin{array}{l}\text { Dactyloctenium aegypticum (L.) } \\
\text { Gaerth }^{\text {*\# }}\end{array}$ & Makure jhar & Poaceae & - & - & + \\
\hline 25. & 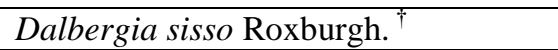 & Sisam & Leguminosae & + & + & + \\
\hline 26. & Desmodium triflorum (L.) DC. ${ }^{\dagger}$ & Bute kanike & Fabaceae & + & + & + \\
\hline 27. & Digitaria sanguinalis (L.) Scop. ${ }^{* \#}$ & Saano banso & Poaceae & - & - & + \\
\hline 28. & $\begin{array}{l}\text { Dioscorea deltoidea } \\
\text { (risebach }\end{array}$ Wallich ex & Bhyakur & Dioscoriaceae & - & + & - \\
\hline 29. & Eclipta prostrata $\mathrm{L}^{* \#}$ & Bhringraj & Asteraceae & - & - & + \\
\hline 30. & Eleusine indica (L.) Gaerth ${ }^{* \#}$ & Kode jhar & Poaceae & - & - & + \\
\hline 31. & Eragrotis tenella (Retz.) Stapf ${ }^{*}$ & Charidana & Poaceae & - & - & + \\
\hline 32. & $\begin{array}{l}\text { Eragrotis unioloids (Retz.) Nees ex } \\
\text { steud. }\end{array}$ & Banso & Poaceae & - & + & + \\
\hline 33. & Euphorbia hirta L. ${ }^{\dagger}$ & Dudhe & Euphorbiaceae & + & + & + \\
\hline 34. & Evolvulus nummularius (L.) L & Balu jhar & Convolvulaceae & - & + & + \\
\hline 35. & Dryopteris spp. L. $^{\dagger}$ & Liudo & Polypodiaceae & + & + & + \\
\hline 36. & Gnophalium pensylvanicum Willd ${ }^{* \#}$ & Gubo ghans & Asteraceae & - & - & + \\
\hline 37. & Hemigraphis repanda (L.) Hallierf. ${ }^{*}$ & Ban-pan & Acanthaceae & - & - & + \\
\hline 38. & Imperata cylindrica (L.).P.Beuv. ${ }^{\dagger}$ & Siru & Gramineae & + & + & + \\
\hline 39. & Justicia procumbens L. $^{\dagger}$ & Phool jhar & Acanthaceae & + & + & + \\
\hline 40. & Lantana camara (L.) Moldenke. ${ }^{\dagger}$ & Ban phaanda & Verbenaceae & + & + & + \\
\hline 41. & $\begin{array}{lll}\begin{array}{l}\text { Lindernia } \\
\text { Borbas }\end{array}{ }^{* \#} & \text { procumbens } & \text { (Krock.) } \\
\end{array}$ & Pitamari jhar & Scrophulariaceae & - & - & + \\
\hline 42. & Lippia nudiflora (L.) Rich & Bukkan & Verbenaceae & - & - & + \\
\hline 43. & Ludwigia perennis L. ${ }^{* \#}$ & Lwang jhar & Onagraceae & - & - & + \\
\hline 44. & Marselia minuta $\mathrm{L}^{\dagger}$ & Chaupatay & Marsilaceae & + & + & + \\
\hline 45. & $\begin{array}{l}\text { Mecardonia procumbens } \\
\text { Small }^{* \#}\end{array}$ & Malati jahar & Scrophulariaceae & - & - & + \\
\hline 46. & Melia azedarach $\mathrm{L}^{\#}$ & Bakaino & Meliaceae & + & - & - \\
\hline 47. & Mimosa pudica L. ${ }^{* \#}$ & Lajjawati & Leguminaceae & - & - & + \\
\hline 48. & Morus alba L. ${ }^{\#}$ & Kimu & Moraceae & + & - & + \\
\hline 49. & Murraya koenigii (L) Sprengel ${ }^{*}$ & Curry patta & Rutaceae & - & + & - \\
\hline 50. & Oxalis corniculata $\mathrm{L}^{\dagger}$ & Chari amilo & Oxalidaceae & + & + & + \\
\hline 51. & Panicum decompositum R.Br. & Chhate ghans & Poaceae & - & + & - \\
\hline 52. & Parthenium hysterophorus L. & Bahudal ghans & Asteraceae & - & + & + \\
\hline 53. & Phyllanthus urinaria L. ${ }^{\#}$ & Bhuin amala & Euphorbiaceae & + & - & + \\
\hline 54. & Polygonum plebejum R.Br. ${ }^{\#}$ & Sukul jhar & Polygonaceae & + & - & + \\
\hline 55. & Piper longum $\mathrm{L}^{\dagger}$ & Pipali & Piperaceae & + & + & + \\
\hline 56. & Portulica oleraceae L. ${ }^{* \#}$ & Nundhiki & Portulaceae & - & - & + \\
\hline 57. & Primula veris $(\mathrm{L} .)^{*}$ & Pelo ghans & Primulaceae & - & + & + \\
\hline 58. & Pumia aromatic L.\# & Jhuse ghans & Compositae & + & - & + \\
\hline 59. & Saccharum ravennae L." & Kaans & Poaceae & + & - & + \\
\hline 60. & $\begin{array}{l}\text { Setaria pumila (Poiret) Romer and } \\
\text { Schulets }\end{array}$ & Bhaale banso & Poaceae & - & - & + \\
\hline
\end{tabular}




\begin{tabular}{|c|c|c|c|c|c|c|}
\hline 61. & Solanum nigrum $(\mathrm{L})^{\dagger}$ & Kali kuiyan & Solanaceae & + & + & + \\
\hline 62. & Spilanthus paniculata Wall. ex DC & Sirsire & Asteraceae & + & + & - \\
\hline 63. & Syzygium cumini $(\mathrm{L})$ Skeels ${ }^{\#}$ & Jamun & Myrtaceae & + & - & + \\
\hline 64. & Tridax procumbens. L. & Husure jhar & Asteraceae & + & - & + \\
\hline 65. & Urena sinutate L. ${ }^{* \#}$ & Kuro & Malvaceae & - & - & + \\
\hline 66. & Xanthium strumerium $\mathrm{L}^{*}$ & Bhende kure & Compositae & - & + & + \\
\hline 67. & Ziziphus jujube (L) Gaertner* & Bayer & Rhamnaceae & - & + & + \\
\hline
\end{tabular}

absent at site $1,{ }^{\#}$ absent at site $2,{ }^{* *}$ absent at site 3 and ${ }^{\dagger}$ common at all sites.

\section{Composition of plant Species}

In the present finding, a total number of 67 plant species including Piper longum were recorded in different study sites. The total density of plant species was 144.25 individuals $\mathrm{m}^{-2}$ at site $1,156.33$ individuals $\mathrm{m}^{-2}$ at site 2 and 237.6 individuals $\mathrm{m}^{-2}$ at site 3 . The density of species at site 3 (237.64) revealed that it had more plant species than that of site 1 and site 2 . At site 1 highest density was contributed by Imperata cylindrica $\left(15.67\right.$ individuals $\left.\mathrm{m}^{-2}\right)$ and lowest was that of Azadirachta indica, Dalbergia sisso and Morus alba having 0.33 individuals $\mathrm{m}^{-2}$ whereas at site 2, the highest density of Commelina paludosa (12.67 individuals $\left.\mathrm{m}^{-2}\right)$ and lowest by Azadirachta indica, $(0.33$ individuals $\mathrm{m}^{-2}$ ) and site 3 highest density was contributed by Parthenium hysterophorus (16.67 individiuals $\mathrm{m}^{-2}$ ) and lowest was Acacia catechu $\left(0.32\right.$ individuals $\left.\mathrm{m}^{-2}\right)$.

In the present study, the maximum and minimum IVI was occupied by Cynodon dactylon (24.49) and Acacia catechu (3.95) species respectively at site 1, the maximum and minimum IVI was occupied by Cynodon dactylon (23.11) and Chenopodium ficifolium (4.01) and Azadirachta indica (4.01) species respectively at site 2, and the maximum and minimum IVI was occupied by Imperata cylindrica (22.87) and Acacia catechu (3.39) species, respectively at site 3 (Table 2).

Table 2:-Composition of different plant species associated with Piper longum at different study sites with their density and Importance Value Index (IVI).

\begin{tabular}{|c|c|c|c|c|c|c|c|c|}
\hline \multirow[t]{3}{*}{ S.No. } & \multirow[t]{3}{*}{ Name of plant species } & \multirow[t]{3}{*}{ Family } & \multicolumn{6}{|c|}{ Distribution of plant species at different sites } \\
\hline & & & \multicolumn{2}{|c|}{ Site 1} & \multicolumn{2}{|c|}{ Site 2} & \multicolumn{2}{|c|}{ Site 3} \\
\hline & & & $\mathbf{D}$ & IVI & D & IVI & D & IVI \\
\hline 1. & Acacia catechu (L.) Willd ${ }^{\#}$ & Leguminosae & 0.50 & 3.95 & - & - & 0.32 & 3.39 \\
\hline 2. & Achyranthes aspera L. $^{\dagger}$ & Amaranthaceae & 1.2 & 5.68 & 0.67 & 5.54 & 1.6 & 4.49 \\
\hline 3. & Ageratum conyzoids L. ${ }^{\dagger}$ & Asteraceae & 6.4 & 23.42 & 4.67 & 15.51 & 6.1 & 18.45 \\
\hline 4. & $\begin{array}{l}\text { Alternanthera sessilis (L.) } \\
\text { DC. }{ }^{* \#}\end{array}$ & Amaranthaceae & - & - & - & - & 12.33 & 15.38 \\
\hline 5. & $\begin{array}{l}\text { Alysicarpus vaginalis (L.) } \\
\text { DC. }{ }^{* \#}\end{array}$ & Leguminaceae & - & - & - & - & 1.0 & 4.49 \\
\hline 6. & Amaranthus spinosus L. ${ }^{*}$ & Amaranthaceae & - & - & 11.33 & 15.14 & 0.67 & 4.28 \\
\hline 7. & Amaranthus viridus L. ${ }^{* \#}$ & Amaranthaceae & - & - & - & - & 2.1 & 5.81 \\
\hline 8. & Argemone mexicana L. ${ }^{*}$ & Papaveraceae & - & - & 1.2 & 5.84 & 1.33 & 5.38 \\
\hline 9. & Asparagus recemosus Willd & Liliaceae & 1.3 & 5.68 & - & - & 0.67 & 4.28 \\
\hline 10. & $\begin{array}{l}\text { Azadirachta indica (Aiton) } \\
\text { Dryand } .^{\dagger}\end{array}$ & Meliaceae & 0.33 & 3.96 & 0.33 & 4.01 & 0.33 & 3.39 \\
\hline 11. & Blumea aromatica $\mathrm{L}^{\dagger}$ & Asteraceae & 0.67 & 6.61 & 0.67 & 5.54 & 1.2 & 4.49 \\
\hline 12. & Brachieria ramosa (L.) Stapf. & Poaceae & 9.67 & 19.22 & 8.67 & 17.43 & 7.4 & 16.5 \\
\hline 13. & Calotropis procera Juss. ${ }^{*}$ & Asclepiadaceae & - & - & - & - & 0.67 & 4.28 \\
\hline 14. & Cassia tora L. ${ }^{\dagger}$ & Fabaceae & 1.69 & 7.42 & 1.4 & 5.84 & 1.33 & 5.38 \\
\hline 15. & Centella asiatica (L.)Urban. & Umbelliferae & - & - & 1.67 & 7.68 & 11.0 & 17.49 \\
\hline 16. & 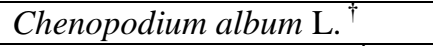 & Amaranthaceae & 0.67 & 5.42 & 0.35 & 4.01 & 1.3 & 5.17 \\
\hline 17. & Cisssampelos pareira $\mathrm{L} .^{\dagger}$ & Menispermaceae & 2.4 & 7.68 & 3.34 & 9.2 & 3.35 & 6.66 \\
\hline 18. & $\begin{array}{l}\text { Clerodendrum indicum (L) } \\
\text { Kuntce. }{ }^{\dagger}\end{array}$ & Labiatae & 3.33 & 8.76 & 2.67 & 8.59 & 4.0 & 7.09 \\
\hline
\end{tabular}




\begin{tabular}{|c|c|c|c|c|c|c|c|c|}
\hline 19. & Commelina benghalensis (L) & Commelinaceae & 5.2 & 15.49 & 2.2 & 6.76 & 2.33 & 5.34 \\
\hline 20. & 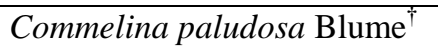 & Commelinaceae & 1.67 & 7.42 & 12.67 & 15.54 & 12.34 & 18.38 \\
\hline 21. & Cynodon dactylon (L) Pers. $^{\dagger}$ & Gramineae & 7.73 & 24.49 & 8.33 & 23.11 & 8.67 & 22.57 \\
\hline 22. & Cyperus esculentus L." & Cyperaceae & 1.33 & 5.95 & - & - & 13.68 & 19.92 \\
\hline 23. & Cyperus rotundus L. ${ }^{\dagger}$ & Cyperacera & 11.0 & 20.68 & 12.67 & 21.46 & 13.0 & 19.49 \\
\hline 24. & $\begin{array}{l}\text { Dactyloctenium aegypticum } \\
\text { (L.) Gaerth }{ }^{* \#}\end{array}$ & Poaceae & - & - & - & - & 10.1 & 15.17 \\
\hline 25. & Dalbergia sisso Roxburgh. $^{\dagger}$ & Leguminosae & 0.33 & 3.96 & 1.2 & 5.84 & 0.87 & 4.28 \\
\hline 26. & $\begin{array}{l}\text { Desmodium triflorum (L.) } \\
\text { DC. }^{\dagger}\end{array}$ & Fabaceae & 3.33 & 8.76 & 3.33 & 6.46 & 2.33 & 6.02 \\
\hline 27. & $\begin{array}{l}\text { Digitaria sanguinalis } \quad \text { (L.) } \\
\text { Scop. }{ }^{*}\end{array}$ & Poaceae & - & - & - & - & 0.66 & 4.28 \\
\hline 28. & $\begin{array}{l}\text { Dioscorea deltoidea Wallich } \\
\text { ex Grisebach }\end{array}$ & Dioscoriaceae & - & - & 1.1 & 5.84 & - & - \\
\hline 29. & Eclipta prostrata L. $^{* \#}$ & Asteraceae & - & - & - & - & 1.35 & 5.38 \\
\hline 30. & Eleusine indica (L.) Gaerth ${ }^{* \#}$ & Poaceae & - & - & - & - & 1.49 & 5.38 \\
\hline 31. & $\begin{array}{lll}\text { Eragrotis } & \text { tenella } & \text { (Retz.) } \\
\text { Stapf }^{* \#}\end{array}$ & Poaceae & - & - & - & - & 1.36 & 5.38 \\
\hline 32. & $\begin{array}{l}\text { Eragrotis unioloids (Retz.) } \\
\text { Nees ex steud. }\end{array}$ & Poaceae & - & - & 0.67 & 5.54 & 2.2 & 5.81 \\
\hline 33. & Euphorbia hirta L. ${ }^{\dagger}$ & Euphorbiaceae & 8.2 & 15.03 & 11.67 & 18.18 & 10.33 & 15.91 \\
\hline 34. & $\begin{array}{l}\text { Evolvulus nummularius (L.) } \\
\mathrm{L}\end{array}$ & Convolvulaceae & - & - & 2.33 & 7.06 & 1.77 & 4.92 \\
\hline 35. & Dryopteris spp. L. $^{\dagger}$ & Polypodiaceae & 1.67 & 7.42 & 1.68 & 7.68 & 2.33 & 6.02 \\
\hline 36. & $\begin{array}{l}\text { Gnophalium pensylvanicum } \\
\text { Willd }{ }^{* \#}\end{array}$ & Asteraceae & - & - & - & - & 1.4 & 4.28 \\
\hline 37. & $\begin{array}{l}\text { Hemigraphis repanda (L.) } \\
\text { Hallierf. }\end{array}$ & Acanthaceae & - & - & - & - & 1.1 & 4.49 \\
\hline 38. & $\begin{array}{l}\text { Imperata cylindrica (L.). P. } \\
\text { Beuav. }^{\dagger}\end{array}$ & Graminae & 15.67 & 21.24 & 11.0 & 19.73 & 15.33 & 22.87 \\
\hline 39. & Justicia procumbens L. $^{\dagger}$ & Acanthaceae & 2.7 & 7.68 & 1.36 & 6.14 & 1.67 & 4.92 \\
\hline 40. & $\begin{array}{l}\text { Lantana camara } \\
\text { Moldenke. }{ }^{\dagger}\end{array}$ & Verbenaceae & 1.2 & 5.68 & 1.33 & 6.14 & 1.68 & 4.92 \\
\hline 41. & $\begin{array}{l}\text { Lindernia procumbens } \\
\text { (Krock.) Borbas }\end{array}$ & Scrophulariaceae & - & - & - & - & 1.67 & 4.92 \\
\hline 42. & Lippia nudiflora (L.) Rich & Verbenaceae & - & - & - & - & 2.33 & 6.02 \\
\hline 43. & Ludwigia perennis L. ${ }^{* \#}$ & Onagraceae & - & - & - & - & 1.33 & 4.7 \\
\hline 44. & Marselia minuta $\mathrm{L}^{\dagger}$ & Marsilaceae & 2.33 & 7.95 & 3.34 & 9.2 & 3.2 & 6.45 \\
\hline 45. & $\begin{array}{l}\text { Mecardonia procumbens } \\
\text { (Miller) Small }^{\text {*\# }}\end{array}$ & Scrophulariaceae & - & - & - & - & 1.3 & 4.49 \\
\hline 46. & Melia azedarach L. ${ }^{\#}$ & Meliaceae & 1.3 & 5.68 & - & - & - & - \\
\hline 47. & Mimosa pudica L. ${ }^{* \#}$ & Leguminaceae & - & - & - & - & 2.33 & 6.02 \\
\hline 48. & Morus alba L." & Moraceae & 0.33 & 3.96 & - & - & 0.67 & 4.28 \\
\hline 49. & $\begin{array}{lll}\text { Murraya } & \text { koenigii } \\
\text { Sprengel }^{*}\end{array}$ & Rutaceae & - & - & 0.69 & 4.32 & - & - \\
\hline 50. & Oxalis corniculata $\mathrm{L}^{\dagger}$ & Oxalidaceae & 7.33 & 17.92 & 8.67 & 18.26 & 10.67 & 19.12 \\
\hline 51. & $\begin{array}{l}\text { Panicum decompositum } \\
\text { R.Br. }\end{array}$ & Poaceae & - & - & 0.68 & 4.32 & - & - \\
\hline 52. & Parthenium hysterophorus L. & Asteraceae & - & - & 12.1 & 17.98 & 16.67 & 21.88 \\
\hline 53. & Phyllanthus urinaria L." & Euphorbiaceae & 2.33 & 7.95 & - & - & 2.67 & 6.24 \\
\hline 54. & Polygonum plebejum R. Br. ${ }^{\#}$ & Polygonaceae & 0.67 & 4.23 & - & - & 1.1 & 4.49 \\
\hline
\end{tabular}




\begin{tabular}{|c|c|c|c|c|c|c|c|c|}
\hline 55. & Piper longum L. ${ }^{\dagger}$ & Piperaceae & 31.0 & 32.63 & 15.33 & 32.7 & 16.33 & 27.46 \\
\hline 56. & Portulica oleraceae L. ${ }^{* \#}$ & Portulaceae & - & - & - & - & 0.67 & 3.6 \\
\hline 57. & Primula veris (L.) ${ }^{*}$ & Primulaceae & - & - & 0.67 & 5.54 & 1.0 & 4.49 \\
\hline 58. & Pumia aromatic $\mathrm{L}^{\#}$ & Asteraceae & 1.0 & 5.68 & - & - & 0.67 & 3.6 \\
\hline 59. & Saccharum ravennae L. ${ }^{\#}$ & Poaceae & 1.6 & 5.68 & - & - & 1.33 & 5.38 \\
\hline 60. & $\begin{array}{l}\text { Setaria pumila (Poiret) } \\
\text { Romer and Schulets }\end{array}$ & Poaceae & - & - & - & - & 0.67 & 4.28 \\
\hline 61. & Solanum nigrum $(\mathrm{L})^{\dagger}$ & Solanaceae & 2.33 & 7.95 & 2.67 & 8.59 & 4.67 & 7.52 \\
\hline 62. & $\begin{array}{l}\text { Spilanthus paniculata Wall. } \\
\text { ex DC }\end{array}$ & Compositae & 0.67 & 5.42 & 0.67 & 7.98 & - & - \\
\hline 63. & Syzygium cumini (L) Skeels ${ }^{\#}$ & Myrtaceae & 1.5 & 5.68 & - & - & 0.33 & 3.39 \\
\hline 64. & Tridax procumbens. L. & Asteraceae & 3.67 & 9.03 & - & - & 2.33 & 5.34 \\
\hline 65. & Urena sinutate L. ${ }^{* \#}$ & Malvaceae & - & - & - & - & 0.67 & 4.28 \\
\hline 66. & Xanthium strumerium $\mathrm{L}^{*}$ & Asteraceae & - & - & 2.33 & 8.28 & 1.67 & 4.92 \\
\hline 67. & Ziziphus jujube (L) Gaertner & Rhamnaceae & - & - & 0.67 & 5.54 & 0.67 & 4.28 \\
\hline \multicolumn{3}{|r|}{ Total } & 144.25 & - & 156.33 & - & 237.64 & - \\
\hline
\end{tabular}

Thus, on the basis of density and importance value index, 16 species of plants including Piper longum were recorded as dominant in all study sites (Table 2). Among them Ageratum conyzoids, Alternanthera sessilis, Brachieria ramosa, Imperata cylindrica, Parthenium hysterophorus, Cyperus rotundus, Cyperus esculentus, and Cynodon dactylon were reported as the world worst weeds in the world by Holm et al. (1977). Of the 16 most important species Brachieria ramosa, Cynodon dactylon, Cyperus rotundus, Euphorbia hirta, Imperata cylindrica and Oxalis corniculata were common at site 1 , site 2 and site 3 .

\section{Eco-phenological Study of Piper longum \\ Plant height}

Piper longum is a perennial herb. The flowering stage started from July-August and turned into fruiting stage in October-January. The harvesting was done in the month of November-January.

The height of the plant was varied among three different sites, i.e. site 1, site 2 and site 3 .The average height of the plant at site 1 was found to be $108.1 \pm 5.71 \mathrm{~cm}, 104.2 \pm 5.84 \mathrm{~cm}$ was recorded at site 2 and the average height of plant recorded at site 3 was $101.8 \pm 3 \mathrm{~cm}$ which was minimum among all three sites (Fig. 2).

\section{Leaf area and Circumference of stem}

Leaves of Piper were found to be broadly ovate, entire, glabrous and sub-acute with reticulate venation. They were dark green and shiny. The leaf area was recorded at the flowering stage of Piper. Ten plants were collected and their leaves were measured and average leaf area was calculated. The maximum leaf area was found at the site 1, i.e. $53.92 \pm 3.46 \mathrm{~cm}^{2}$, and then $50.43 \pm 2.69 \mathrm{~cm}^{2}$ at site 2 and minimum leaf area was recorded from the site 3 , i.e. $49.43 \pm 3.61 \mathrm{~cm}^{2}$. Similarly, the mean leaf length and breadth ratio was recorded as $1.01,0.93$ and 0.92 at site 1 , site 2 and site 3, respectively (Fig. 3). The circumference of stem of the Piper was found to be different among the different sites. The mean of the circumference of the stem was found to be $1.0 \pm 0.83 \mathrm{~cm}$ at site $1,0.9 \pm 0.73 \mathrm{~cm}$ at site 2 and at site 3 it was reported as $0.83 \pm 0.38 \mathrm{~cm}$ (Fig.4).

Fig. 2:- Plant height $(\mathrm{cm})$ of Piper in different study sites $($ mean \pm SE).

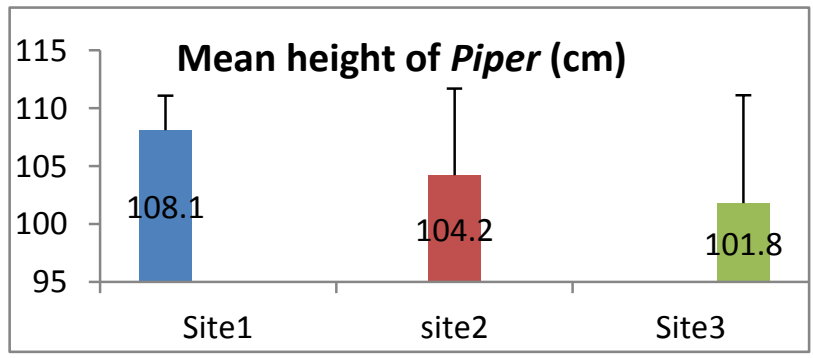

Fig.3:-Leaf area $\left(\mathrm{cm}^{2}\right)$ of Piper in different study sites (mean \pm SE).

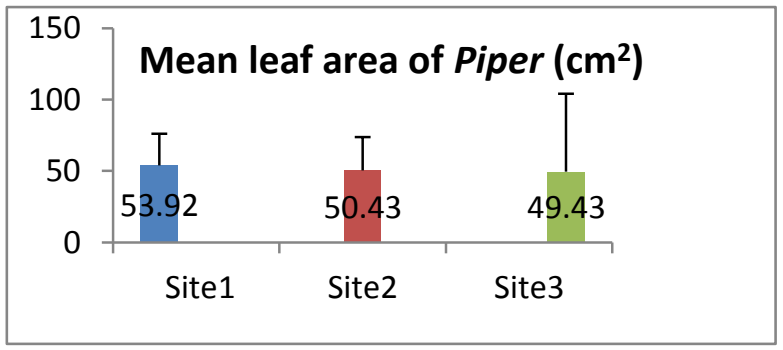




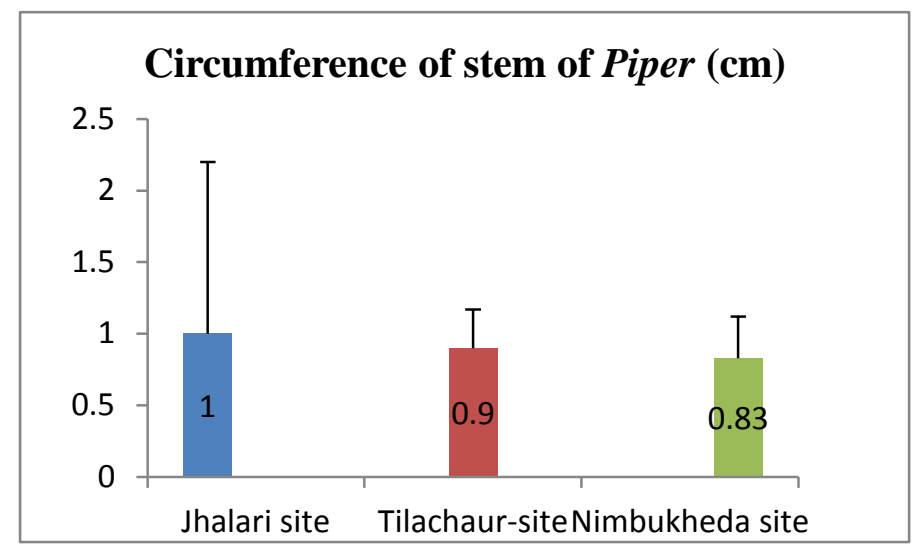

Fig. 4:-Circumference $\left(\mathrm{cm}^{2}\right)$ of Piper of different study sites (mean \pm SE)

\section{Reproductive Behaviours}

Fruits were cylindrical, short and stout. They were shiny dark green when immature and turned darker at maturity. The average sizes of fruits taken from different sites i.e. site 1 , site 2 , and site 3 were found to be $1.79 \pm 0.122$, $1.79 \pm 0.095$ and $1.65 \pm 0.08$, respectively. The average size of 20 seeds of Piper longum was $1.21 \pm 0.016 \mathrm{~mm}$. Single spike of Piper produced $71.33 \pm 1.49,67.67 \pm 1.60$ and $61.33 \pm 1.93$ average seed number at site 1 , site 2 , and site 3 , respectively. The seed output per plant at site 1, site 2 and site 3 was found to be 827.42, 581.96 and 447.709 , respectively.

\section{Medicinal Value of Piper longum}

Plants have been source of medicine for thousands of years. Piper longum is among the important medicinal plants used in various system of medicine. P. longum is a medicinal plant that has been used as therapeutic agent in the treatment of various pathological conditions. The specific effect of plant makes it more useful for animals and human beings. Because Piper longum is widely used in cooking and traditional medicine, it is generally assumed to be safe in moderate doses. The roots and fruits of Piper are generally used to cure different diseases. Leaves of Piper are used as hair tonic. Leaves are used to get rid of lice and dandruff. The root has a bitter, hot and sharp taste. It is used as a carminative, a tonic to liver, stomachic, abortifacient, emmenagogue, haematinic, diuretic, digestive and as a general tonic, useful in inflammation of liver, pains in joints, lumbago, snake bite, scorpion-sting and night blindness. A decoction of roots is given for swelling of joints of cattle.

The major alkaloid of long pepper is piperine. Piperine is antipyretic (reduces fever), hypotensive (lowers BP), restorative, liver protecting, CNS stimulant and improves drug availability. The roots are known as Pippalimula. The dried, immature, catkin-like fruits of the plant are known as Pippali. The unripe fruit is sweetish, cooling and useful in biliousness. The ripe fruit is sweet, pungent, a stomachic, aphrodisiac, alternative, laxative, antidiarrhoeic, antidysentric, is useful in asthma, bronchitis, abdominal complaints, fevers, leucoderma, urinary discharges, tumors, piles, diseases of the spleen, pains, inflammation, leprosy, epilepsy, insomnia, jaundice, hiccups, tuberculosis and reduces biliousness. The essential oil of Piper longum also shows antibacterial and antifungal action. Piper also helps to deal with indigestion, as it cleans the lining of the intestine, it helps to improve appetite. Piper is also used to treat menstrual cramps, infertility and libido, intellect and memory power. Piper longum is evenly used in both ayurvedic and allopathic medicinal values.

\section{Discussion:-}

In the present study, attempts were made to examine the plant species composition with Piper longum on their growth among the different sites of Kanchanpur, Far Western Nepal. It is used in over 320 classical compound, medicinal formulations and in many modern herbal formulations (Ravindran and Balachandran, 2005). Besides fruits, the roots and thicker parts of stem are cut and dried and used as an important drug in the Ayurvedic and Unani systems (Ali et al., 2007). Phoboo, Devkota and Jha (2008) studied the various aspects of medicinal plants of Nepal and its conservation efforts in context to Nepal. Piper mostly grows in hot and humid climatic conditions. It preferred well drained sandy soil rich in organic matter with $\mathrm{p}^{\mathrm{H}}$ 5.5-8.5 (Sumy et al., 2000). 
The height of Piper was varied among three different sites. The average height of plant was recorded from range $102-108 \mathrm{~cm}$. Minimum height was recorded from Nimbukheda-site due to the presence of associated species which inhibit the growth of the plant to some extent. The average leaf area was found to be $7.33 \mathrm{~cm}^{2}, 6.76 \mathrm{~cm}^{2}$ and 6.9 $\mathrm{cm}^{2}$ at site 1 , site 2 and site 3, respectively. This value matches with the study of Kritikar and Basu (1935) who found the size of leaves as $6.0-9.0 \mathrm{~cm}^{2}$ in India. The average sizes of fruits taken from different sites i.e. site 1, site 2, and site 3 were found to be $1.79 \mathrm{~cm}, 1.79 \mathrm{~cm}$ and $1.65 \mathrm{~cm}$, respectively. The average size of 20 seeds of Piper longum was $1.21 \pm 0.016 \mathrm{~mm}$, the shape of seed was oblong, and the colour was brown. The present distribution of Piper in Nepal was found in tropical regions at altitudes ranging from 100 to $1000 \mathrm{~m}$ from sea level. The study site of present work also lies between the altitudes of this range. The growth and reproduction cycle of Piper depends on climatic conditions such as humidity, available moisture, soil $\mathrm{p}^{\mathrm{H}}$ and perennial life cycle. In the present study, the distribution pattern is more or less similar with the finding of Baral and Kurmi (2006).

The composition of plant species with Piper was varied among the different sites. In the present work 67 plant species from different 34 families including Piper longum were studied in all study sites of Kanchanpur. Among them $36(53.73 \%)$ species of plant with Piper were recorded from Jhalari-site. Similarly, $37(55.22 \%)$ and 62 (92.53\%) plant species were recorded among Tilachaur-site and Nimbukheda-site, respectively. The composition of different species with Piper indicates the poor growth of Piper longum due to space, nutrient deficiency, light intensity, public involvement, cattle grazing, fodder collection, climatic conditions etc.

On the basis of density and IVI, 16 plant species including Piper were recorded as dominant (IVI >15) among study sites. Ageratum conyzoids (23.42) Brachieria ramosa (19.22) Commelina benghalensis (15.49), Cynodon dactylon (19.7), Cyperus rotundus (20.68), Euphorbia hirta (15.03), Imperata cylindrica (21.22), Oxalis corniculata (17.92), and Piper longum (32.63) were dominant at site 1. Ageratum conyzoids (15.51), Amaranthus spinosus (15.14), Brachieria ramosa (17.43), Commelina paludosa (15.54), Cynodon dactylon (23.11), Cyperus rotundus (21.46, Euphorbia hirta (18.18), Imperata cylindrica (19.73), Oxalis corniculata (18.26), Parthenium hysterophorus (17.98) and Piper longum (32.7) species were dominant at site 2 and Ageratum conyzoids (18.45), Alternanthera sessilis, Brachieria ramosa (16.5), Centella asiatica (17.49), Commelina paludosa (18.38), Cynodon dactylon (22.57), Cyperus rotundus (19.49), Dactyloctenium aegyptium (15.17), Euphorbia hirta (18.6), Imperata cylindrica (22.87), Oxalis corniculata (19.12), Evolvulus nummularis (46.6), Parthenium hysterophorus (21.88) and Piper longum (27.46) species at site 3 .

Among them Ageratum conyzoids, Alternanthera sessilis, Brachieria ramosa, Imperata cylindrica, Parthenium hysterophorus, Cyperus rotundus, Cyperus esculentus, and Cynodon dactylon are reported as being the important species in the world by Holm et al. (1977). Of the 16 most important species Brachieria ramosa, Cynodon dactylon, Cyperus rotundus, Euphorbia hirta, Imperata cylindrica and Oxalis corniculata were common at site 1, site 2 and site 3.

Density of Piper longum was found to be $31.0 \mathrm{~m}^{-2}, 15.33 \mathrm{~m}^{-2}$ and $16.33 \mathrm{~m}^{-2}$ at Jhalari-site, Tilachaur-site and Nimbukheda-site, respectively. It might be due to the soil composition, humidity and favorable weather conditions that the density was highest in Jhalari-site.

Piper was used as medicine for both animal and human beings. According to Iyer (1983) it is also reported that pipali cures cough dyspnoea, ascites, leprosy, diabetes, piles, colic indigestion, anemia, thirst and dispels cardiac and splenic disorder, fever, loss of appetite and worm troubles. CSIR (1998) reported the medicinal uses of pipali as for diseases of respiratory tract, cough and bronchitis, asthma etc. In present work by interviewing with several related core groups, was found that Piper used in cough, common cold, fever, scorpion-sting and snake bite etc. This result was more or less similar to that of Chahal et al., (2011) who reported that the roots and fruits of the plant are used as an antidote to snake bite, scorpion stings, chronic bronchitis, cough and cold and the ripe fruits are used as an alternative to tonic.

\section{Acknowledgement:-}

The author is thankful to Campus Chief, Siddhanath Science Campus, Tribhuvan University Mahendranagar, Nepal, for providing facilities throughout the study period. 


\section{References:-}

1. Akiyama, S.K. Yonekura and H. Ohba 1998. New Records and treatment of Nepalese Flowering Plants. Newsletter of Himalaya Botany, No. 23: 18-25.

2. Ali, M.A., Alam, N.M., Yeasmin, M.S., Khan, A.M. and Sayeed, M.A. 2007. Antimicrobial screening of differential extracts of Piper longum L. Res. J. Agric. Biol. Sci. 3(6): 852-857.

3. Anshuman P.S., Singh K.P. and Aasra K.G. 1984. Effect of Vardhaman Pippali (Piper longum) on Patients with respiratory disorders, Sachitra Ayurv, 37(1): 47-49.

4. Banergji, M.L. 1963. Some edible and medicinal Plants from east Nepal. Journal of the Bombay Natural History Society, 53:153-155.

5. Banerjee, N.S., Manoj P. and Das M.R. 1999. Male sex associated RAPD makers in Piper longum Linn. Curr Sci, 77(5): 693-695.

6. Baral, S.R. and P.P. Kurmi 2006. A Compendium of Medicinal Plants in Nepal. Mass printing press, Kathmandu, Nepal.

7. Chahal, J., Ohlyan, R., Kandale, A., Walia, A. and Puri, S. 2011. Introduction, phytochemistry, traditional uses and biological activity of genus Piper: a review. Intl. J. Curr. Pharm. Revw. Res. 2(2):130-144.

8. CSIR, 1998. The Wealth of India: Raw materials Vol. I. (Revised edition). Publications and Information Directorate, New Delhi, 31-32 pp.

9. Curtis, J.T, and Mclntosh 1951. An upland forest continuum in the Prarie Forest border region of Wisconsin. Ecology, 32: 476-96.

10. Dahanukar S.A., Karandikar S.M. and Desai M. 1984. Efficacy of Piper longum in childhood asthma, Indian Drugs, 21(9): 384-388.

11. Doberemez Z.J.F. 1976. Le Nepal ecological et biogeographic, Paris Centre National de la Recherche Scientdifique.

12. Holm, G.L., D.L. Plucknett, J.V. Pancho and J.P. Herberger 1977. The world's worst weeds. Distribution and biology. East west center press, Honolulu, Hawaii, 609 pp.

13. Iyer, S.R. 1983. Evaluation of Liver protective potential of Piperine. Yogasamgraham, Aryavaidyasala, Kottakkal, 24pp.

14. Kirtikar K.R., Basu B.D. 1980. Indian Medicinal Plants. Mumbai, India: Orients Longman: 21-28.

15. Koba, H.S., Akiyama, Y. Endo and H. Ohba 1994. List of Flowering Plants and Gymnosperms of Nepal. The University Museum, University of Tokyo.

16. Misra, R. 1968. Ecology Workbook. Oxford and IBH Publishing Co., New Delhi, India.

17. Phaboo, S.A. Devkota and P.K. Jha 2008. Medicinal Plants in Nepal- An overview. Medicinal plant in Nepal, An Anthology of contemporary Research, Ecological Society (Ecos) 1-24.

18. Rastogi R.P., Malhotra B.N. 1993. Compendium of Indian Medicinal Plants. CDRI, Lucknow and New Delhi, India. Nisc: $504-857$.

19. Ravindran, P. N. and Balachandran, I. 2005. Thippali-the Indian long pepper-The most widely used medicinal plant of India. Spice India 18 (11): 32-42.

20. Salisbury, E.J. 1942. The reproductive studies of plant studies in quantitive biology. G. Bells and Sons, London, UK.

21. Stainton, J.D.A. 1972. Forest of Nepal, Heffner Publishing Company, New York.

22. Stearn, W.T. 1960. Allium and Milula in the Central and Eastern Himalaya, Bull. Br. Mus. Nat. Hist. (Bot) 2:159-191.

23. Sumy Oommen, Ved D.K. and Krishan R. 2000. Tropical Indian Medicinal plants, propagation methods, 268269.

24. Viswananthan T.V. 1995. Medicinal and Aromatic plants, K.I. Chadha and Gupta (eds.), Malhotra Publishing House, New Delhi, 373-383.

25. Wilson, E.O. 1988. The current state of biological Biodiversity. In: Biodiversity (eds. E.O. Wilson, E.O. and F.M. Peter) National Academy Press, Washington, DC, 3-18 pp.

26. Wilson, E.O. 1992. The Diversity of life. W.W. Norton \& Company, New York, London.

27. Wilson, E.O. 1988. The current state of biological Biodiversity. In: Biodiversity (eds. E.O. Wilson, E.O. and F.M. Peter) National Academy Press, Washington, DC pp. 3-18.

28. Zobal, D.B., P.K. Jha, M.L. Behan and U.R.K. Yadav 1987. A Practical Manual for Ecology. Ratna Book Distributor, Kathmandu, Nepal. 